\title{
REMARKS ON A LIMITING CASE OF HARDY TYPE INEQUALITIES
}

\author{
Megumi Sano and TAKuYa Sobukawa
}

Abstract. The classical Hardy inequality holds in Sobolev spaces $W_{0}^{1, p}$ when $1 \leqslant p<N$. In the limiting case where $p=N$, it is known that by introducing a logarithmic weight function in the Hardy potential, some inequality which is called the critical Hardy inequality holds in $W_{0}^{1, N}$. In this note, in order to give an explanation of the appearance of the logarithmic function in the potential, we derive the logarithmic function from the classical Hardy inequality with best constant via some limiting procedure as $p / N$. We show that our limiting procedure is also available for the classical Rellich inequality in second order Sobolev spaces $W_{0}^{2, p}$ with $p \in\left(1, \frac{N}{2}\right)$ and the Poincaré inequality.

Mathematics subject classification (2010): 35A23, 46B30, 35A08.

Keywords and phrases: Hardy inequality, limiting case, Sobolev embedding, extrapolation, pointwise estimate of radial functions.

\section{REFERENCES}

[1] Adimurthi, K. SandeEP, Existence and non-existence of the first eigenvalue of the perturbed Hardy-Sobolev operator, Proc. Roy. Soc. Edinburgh Sect. A 132 (2002), No. 5, 1021-1043.

[2] Adimurthi, S. SAntra, Generalized Hardy-Rellich inequalities in critical dimension and its applications, Commun. Contemp. Math., 11 (2009), No. 3, 367-394.

[3] A. Alvino, A limit case of the Sobolev inequality in Lorentz spaces, Rend. Accad. Sci. Fis. Mat. Napoli (4) 44 (1977), 105-112 (1978).

[4] P. Baras, J. A. Goldstein, The heat equation with a singular potential, Trans. Amer. Math. Soc., 284 (1984), 121-139.

[5] W. Beckner, And M. Pearson, On sharp Sobolev embedding and the logarithmic Sobolev inequalitiy, Bull. London Math. Soc., 30 (1998), 80-84.

[6] C. Bennett, K. Rudnick, On Lorentz-Zygmund spaces, Dissertationes Math. (Rozprawy Mat.) 175 (1980), $67 \mathrm{pp}$.

[7] C. Bennett, R. Sharpley, Interpolation of Operators, Pure and Applied Mathematics, vol. 129, Boston Academic Press, Inc., (1988).

[8] H. BREZIS, J. L. VÁZQUEZ, Blow-up solutions of some nonlinear elliptic problems, Rev. Mat. Univ. Complut. Madrid 10 (1997), No. 2, 443-469.

[9] X. CABré, X. Ros-Oton, Sobolev and isoperimetric inequalities with monomial weights, J. Differential Equations. 255, (2013), 4312-4336.

[10] L. Carleson, S.-Y. A. Chang, On the existence of an extremal function for an inequality of J. Moser, Bull. Sci. Math. (2) 110 (1986), no. 2, 113-127.

[11] D. CASSANi, B. Ruf, C. TARsi, Best constants for Moser type inequalities in Zygmund spaces, Mat. Contemp. 36 (2009), 79-90.

[12] D. CASSAni, B. RuF, C. TARSi, Best constants in a borderline case of second-order Moser type inequalities, Ann. Inst. H. Poincare Anal. Non Lineaire 27 (2010), no. 1, 73-93.

[13] D. CASSANI, B. RUF, C. TARSI, Equivalent and attained version of Hardy's inequality in $\mathbb{R}^{n}, \mathrm{~J}$. Funct. Anal. 275 (2018), no. 12, 3303-3324.

[14] D. CASSAni, B. RuF, C. TARsi, Group invariance and Pohozaev identity in Moser-type inequalities, Commun. Contemp. Math. 15 (2013), No. 2, 1250054, 20 pp. 
[15] D. CASSANI, B. RUF, C. TARSI, On the capacity approach to non-attainability of Hardy's inequality in $\mathbb{R}^{N}$, Discrete Contin. Dyn. Syst. Ser. S 12 (2019), no. 2, 245-250.

[16] D. CASSANI, F. SANI, C. TARSI, Equivalent Moser type inequalities in $\mathbb{R}^{2}$ and the zero mass case, J. Funct. Anal. 267 (2014), no. 11, 4236-4263.

[17] E. B. Davies, A. M. HinZ, Explicit constants for Rellich inequalities in $L^{p}(\Omega)$, Math. Z. 227 (1998), no. 3, 511-523.

[18] A. Detalla, T. Horiuchi And H. Ando, Missing terms in Hardy-Sobolev inequalities, Proc. Japan Acad. Ser. A Math. Sci. 80 (2004), no. 8, 160-165.

[19] F. Gazzola, H.-C. Grunau, G. Sweers, Polyharmonic boundary value problems. Positivity preserving and nonlinear higher order elliptic equations in bounded domains, Lecture Notes in Mathematics, 1991. Springer-Verlag, Berlin, (2010), xviii+423 pp.

[20] T. Horiuchi, P. Kumlin, On the Caffarelli-Kohn-Nirenberg-type inequalities involving critical and supercritical weights, Kyoto J. Math. 52 (2012), no. 4, 661-742.

[21] N. IoKU, Attainability of the best Sobolev constant in a ball, Math. Ann. 375 (2019), no. 1-2, 1-16.

[22] N. IOKU, M. Is HiwATA, A Scale Invariant Form of a Critical Hardy Inequality, Int. Math. Res. Not. IMRN (2015), no. 18, 8830-8846.

[23] B. KAWOHL, V. FRIDMAN, Isoperimetric estimates for the first eigenvalue of the $p$-Laplace operator and the Cheeger constant, Comment. Math. Univ. Carolin. 44 (2003), no. 4, 659-667.

[24] O. A. LADYZHENSKAYA, The mathematical theory of viscous incompressible flow, Second edition, revised and enlarged, Mathematics and its Applications, Vol. 2, Gordon and Breach, Science Publishers, New York-London-Paris, (1969).

[25] J. LERAY, Etude de diverses equations integrales non lineaires et de quelques problemes que pose l'hydrodynamique (French), (1933), 82 pp.

[26] N. LAm, G. Lu, L. ZhANG, Equivalence of critical and subcritical sharp Trudinger-Moser-Adams inequalities, Rev. Mat. Iberoam. 33 (2017), no. 4, 1219-1246.

[27] E. Mitidieri, A simple approach to Hardy inequalities, (Russian) Mat. Zametki 67 (2000), no. 4, 563-572; translation in Math. Notes 67 (2000), no. 3-4, 479-486.

[28] F. RELLICH, Halbbeschränkte Differentialoperatoren höherer Ordnung, (German) Proceedings of the International Congress of Mathematicians, 1954, Amsterdam, vol. III, pp. 243-250. Erven P. Noordhoff N. V., Groningen; North-Holland Publishing Co., Amsterdam, 1956.

[29] L. SALOFF-Coste, Aspects of Sobolev-type inequalities, London Mathematical Society Lecture Note Series, 289, Cambridge University Press, Cambridge, 2002. x+190 pp.

[30] M. SANo, Explicit optimal constants of two critical Rellich inequalities for radially symmetric functions, arXiv: 2002.04768v2.

[31] M. SANo, Extremal functions of generalized critical Hardy inequalities, J. Differential Equations 267 (2019), no. 4, 2594-2615.

[32] M. SANO, Minimization problem associated with an improved Hardy-Sobolev type inequality, Nonlinear Anal. 200 (2020), 111965, 16 pp.

[33] M. SANO, Two limits on Hardy and Sobolev inequalities, arXiv:1911.04105.

[34] N. S. Trudinger, On imbeddings into Orlicz spaces and some applications, J. Math. Mech. 17 (1967), 473-483.

[35] S. YANO, Notes on Fourier analysis XXIX, An extrapolation theorem, J. Math. Soc. Japan 3, (1951), 296-305.

[36] A. Zygmund, Trigonometric Series, 2nd ed. Vols. I, II, Cambridge University Press, New York 1959 Vol. I. xii+383 pp.; Vol. II. vii+354 pp. 